Claremont Colleges

Scholarship@ Claremont

All HMC Faculty Publications and Research

HMC Faculty Scholarship

$1-1-1980$

\title{
A New Lower Bound for the Number of Switches in Rearrangeable Networks
}

Nicholas Pippenger

Harvey Mudd College

\section{Recommended Citation}

Pippenger, Nicholas. "A New Lower Bound for the Number of Switches in Rearrangeable Networks." SIAM Journal on Algebraic and Discrete Methods 1, no. 2 (June 1980): 164-167.

This Article is brought to you for free and open access by the HMC Faculty Scholarship at Scholarship @ Claremont. It has been accepted for inclusion in All HMC Faculty Publications and Research by an authorized administrator of Scholarship @ Claremont. For more information, please contact scholarship@cuc.claremont.edu. 


\title{
A NEW LOWER BOUND FOR THE NUMBER OF SWITCHES IN REARRANGEABLE NETWORKS*
}

\author{
NICHOLAS PIPPENGER $\dagger$
}

\begin{abstract}
For the commonest model of rearrangeable networks with $n$ inputs and $n$ outputs, it is shown that such a network must contain at least $6 n \log _{6} n+O(n)$ switches. Similar lower bounds for other models are also presented.
\end{abstract}

1. Introduction. The lower bound referred to in the title will be established by modeling a rearrangeable network as a directed graph in which vertices represent wires and edges represent switches. A number of alternative models will be considered later.

A n-network $N=(G, A, B)$ comprises a directed graph $G=(V, E)$, with vertices $V$ and edges $E$, a set $A$ of $n$ distinguished vertices called inputs, and a set $B$, disjoint from $A$, of $n$ distinguished vertices called outputs.

A request for $N$ is an ordered pair $(a, b)$ comprising an input $a$ and an output $b$. An assignment for $N$ is a set of requests for $N$, no two having an input or output in common. $A k$-assignment for $N$ is an assignment containing exactly $k$ requests.

A route in $N$ is a directed path in $G$, starting at an input and ending at an output. A state of $N$ is a set of routes in $N$, no two having a vertex in common. The set of states of $N$ will be denoted $\Omega$. A $k$-state of $N$ is a state of $N$ containing exactly $k$ routes. The set of $k$-states of $N$ will be denoted $\Omega_{k}$.

An assignment is said to be realized by a state if, for every request $(a, b)$ in the assignment, there is a route from $a$ to $b$ in the state. An $n$-network $N$ is an $n$-connector if each of the $n ! n$-assignments for $N$ is realized by some state of $N$.

An $n$-connector must satisfy the lower bound

$$
|E| \geqq 3 n \log _{3} n+O(n)
$$

$(3 / \ln 3=2.730 \cdots)$; this follows from the inequality

$$
|E| \geqq 3 \log _{3}\left|\Omega_{n}\right|
$$

(attributed to R. L. Dobrushin by Bassalygo and Tsybakov [1]), from the obvious inequality

$$
\left|\Omega_{n}\right| \geqq n !
$$

(distinct assignments must be realized by distinct states), and from the estimate

$$
\log n !=n \log n+O(n)
$$

(due to Stirling [7, p. 137]).

The purpose of this note is to derive the improved lower bound

$$
|E| \geqq 6 n \log _{6} n+O(n)
$$

$(6 / \ln 6=3.348 \cdots)$; this will follow from the improved inequality

$$
|E| \geqq 6 \log _{6}\left|\Omega_{n}\right| \text {. }
$$

\footnotetext{
* Received by the editors October 3, 1979, and in revised form October 24, 1979.

$\dagger$ Mathematical Sciences Department, IBM Thomas J. Watson Research Center, Yorktown Heights, New York 10598.
} 
These lower bounds may be compared with the upper bound for $n$-connectors,

$$
|E| \leqq 6 n \log _{3} n+O(n)
$$

$(6 / \ln 3=5.461 \cdots$; see Pippenger and Valiant [5, Remark 2.2.6]).

The qualitative significance of these improvements may be seen by comparing these bounds with the corresponding bounds for $n$-shifters ( $n$-networks that need not have states realizing all $n$ ! assignments, but only the $n$ assignments corresponding to cyclic permutations). The inequality (1) actually follows immediately from the even sharper lower bound for $n$-shifters,

$$
|E| \geqq 3 n \log _{3} n
$$

(see Pippenger and Valiant [5, Corollary 2.2.2]). This may be compared with the upper bound for $n$-shifters,

$$
|E| \leqq 3 n \log _{3} n+O(n)
$$

(see Pippenger and Valiant [5, Remark 2.2.5]). The results of this note thus show that $n$-connectors require more edges than $n$-shifters, a plausible conclusion which was, however, not deducible from previous results.

2. The new lower bound. One may assume, without loss of generality, that no edge is directed into an input or directed out of an output, for no such edge can occur in an $n$-state.

If there is any vertex $v$ in $V-(A \cup B)$ out of which no edge is directed, one may omit $v$ from $V$ and omit each edge of the form $(u, v)$ from $E$. If there is any vertex $v$ in $V-(A \cup B)$ out of which exactly one edge $(v, w)$ is directed, one may omit $v$ from $V$ and replace each edge of the form $(u, v)$ by the edge $(u, w)$ in $E$. In either case one obtains a network with at most as many edges and just as many $n$-states. Thus one may assume, without loss of generality, that at least two edges are directed out of each vertex in $V-(A \cup B)$.

Let $f: B \rightarrow A$ be an arbitrary bijection. Let $G^{*}=\left(V^{*}, E^{*}\right)$ be the directed graph with vertices $V^{*}$ and edges $E^{*}$ obtained from $N$ as follows. Let $V^{*}$ be obtained from $V$ by omitting the vertices in $B$. Let $E^{*}$ be obtained from $E$ by replacing each edge of the form $(v, b)$ in $V \times B$ by the edge $(v, f(b))$ in $V \times A$, and by adding the edge $(v, v)$ for each vertex $v$ in $V-(A \cup B)$.

The edges of the form $(v, v)$ added to $E^{*}$ are directed out of vertices $v$ in $V-(A \cup B)$, and $E$ contains at least two edges directed out of each such vertex. Thus

$$
\left|E^{*}\right| \leqq \frac{3}{2}|E| \text {. }
$$

A set of closed directed paths in $G^{*}$ containing exactly one edge directed into each vertex and one edge directed out of each vertex will be called a circulation in $G^{*}$. The set of circulations in $G^{*}$ will be denoted $\Omega^{*}$. Each $n$-state of $N$ corresponds to a circulation in $G^{*}$ (by replacing edges of the form $(v, b)$ by edges $(v, f(b))$ and adding edges of the form $(v, v)$ as necessary), and distinct $n$-states correspond to distinct circulations. Thus

$$
\left|\Omega^{*}\right| \geqq\left|\Omega_{n}\right| .
$$

By virtue of these inequalities, it will suffice to show

$$
\left|E^{*}\right| \geqq 9 \log _{6}\left|\Omega^{*}\right|
$$

for an arbitrary directed graph $G^{*}$. 
Let $M$ be the $(0,1)$-matrix with rows and columns indexed by $V^{*}$ and with $(v, w)$ th entry $M_{v, w}$ equal to 1 or 0 according as $(v, w)$ does or does not appear in $E^{*}$. Let

$$
L_{v}=\sum_{w \in V^{*}} M_{v, w}
$$

denote the sum of the entries in the $v$ th row of $M$. Then

$$
\left|E^{*}\right|=\sum_{v \in V^{*}} L_{v}
$$

On the other hand,

$$
\left|\Omega^{*}\right|=\operatorname{per} M
$$

where per $M$ denotes the permanent of $M$, since both sides count the number of permutations $g$ of $V^{*}$ for which $(v, g(v))$ appears in $E^{*}$ for each $v$ in $V^{*}$. Thus it will suffice to show

$$
\sum_{v \in V^{*}} L_{v} \geqq 9 \log _{6} \operatorname{per} M
$$

for an arbitrary $(0,1)$-matrix $M$.

The inequality

$$
\sum_{v \in V^{*}}\left(\log \left(L_{v} !\right)\right) / L_{v} \geqq \log \operatorname{per} M
$$

for an arbitrary $(0,1)$-matrix $M$ was conjectured by Minc [3] and proved by Bregman [2] (see Schrijver [6] for a particularly simple and elegant proof). Since the expression $(\log (L !)) / L^{2}$ assumes its maximum over integers $L$ at $L=3$,

$$
\begin{aligned}
\sum_{v \in V^{*}} L_{v} & \geqq\left(3^{2} / \log (3 !)\right) \sum_{v \in V^{*}}\left(\log \left(L_{v} !\right)\right) / L_{v} \\
& \geqq 9 \sum_{v \in V^{*}}\left(\log _{6}\left(L_{v} !\right)\right) / L_{v} \\
& \geqq 9 \log _{6} \operatorname{per} M .
\end{aligned}
$$

This proves (6), and thus establishes (5), (3) and (2) in turn.

3. Other new lower bounds. The argument of this note is easily extended to a number of other models of rearrangeable networks. The most interesting of these is obtained by replacing directed graphs and directed paths by undirected graphs and undirected paths. A directed graph $G=(V, E)$ can be obtained from an undirected graph $G^{\prime}=\left(V^{\prime}, E^{\prime}\right)$ by setting $V=V^{\prime}$ and replacing each undirected edge $\{v, w\}$ in $E^{\prime}$ by a pair of directed edges $(v, w)$ and $(w, v)$, so that

$$
\left|E^{\prime}\right| \geqq \frac{1}{2}|E| \text {. }
$$

In this way, a directed $n$-connector $N=(G, A, B)$ can be obtained from an undirected $n$-connector $N^{\prime}=\left(G^{\prime}, A^{\prime}, B^{\prime}\right)$ by setting $A=A^{\prime}$ and $B=B^{\prime}$. One may assume, without loss of generality, that at least three undirected edges are incident with each vertex in $V^{\prime}-\left(A^{\prime} \cup B^{\prime}\right)$, so that

$$
\left|E^{*}\right| \leqq \frac{4}{3}|E| .
$$

Continuing with the argument of $\S 2$ leads to the lower bound

$$
\left|E^{\prime}\right| \geqq \frac{27}{8} n \log _{6} n+O(n)
$$


$(27 / 8 \ln 6=1.883 \cdots)$ for undirected $n$-connectors. This may be compared with the previous bound

$$
\left|E^{\prime}\right| \geqq \frac{5}{2} n \log _{4} n+O(n)
$$

$(5 / 2 \ln 4=1.803 \cdots)$ which applies even to undirected $n$-shifters (see Pippenger and Valiant [5, Thm. 2.2.3]). No better upper bounds are known for undirected $n$ connectors and $n$-shifters than for their directed counterparts.

Other, even easier, extensions are to consider "single-ended" or "undifferentiated" $n$-connectors in which the $n$ inputs and $n$ outputs are replaced by a single undifferentiated set of $n$ distinguished vertices called "terminals" (this reduces the leading terms of lower bounds by a factor of 2) and to bound $\log |\Omega|$ rather than merely $\log \left|\Omega_{n}\right|$ (this affects only the $O(n)$ terms). These extensions yield improvements of the results in Pippenger [4].

James Shearer, the referee for this paper, has pointed out some improvements to the foregoing results. In the directed case, a vertex $w$ in $V-(A \cup B)$ into which only two edges $(u, w)$ and $(v, w)$ are directed and out of which only two edges $(w, x)$ and $(w, y)$ are directed can be omitted, the edges being replaced by $(u, x),(v, x),(u, y)$ and $(v, y)$. Repeating this transformation as long as possible yields a graph with just as many edges and $n$-states but in which a total of at least five edges are directed into or out of each vertex in $V-(A \cup B)$. This allows (4) to be sharpened to

$$
\left|E^{*}\right| \leqq \frac{7}{5}|E|,
$$

and results in a lower bound of

$$
|E| \geqq \frac{45}{7} n \log _{6} n+O(n)
$$

$(45 / 7 \ln 6=3.587 \cdots)$. Similarly, in the undirected case, a vertex $w$ in $V-(A \cup B)$ incident with only three edges $\{w, x\},\{w, y\}$ and $\{w, z\}$ can be omitted, the edges being replaced by $\{x, y\},\{y, z\}$ and $\{z, x\}$. This yields a graph in which every vertex in $V-(A \cup B)$ is incident with at least four edges, allows (7) to be sharpened to

$$
\left|E^{*}\right| \leqq \frac{5}{4}|E|
$$

and results in a lower bound of

$$
\left|E^{\prime}\right| \geqq \frac{18}{5} n \log _{6} n+O(n)
$$

$(18 / 5 \ln 6=2.009 \cdots)$

\section{REFERENCES}

[1] L. A. BASSAlygo AND B. S. TsyBAKov, Blocking probability for a rearrangeable switching system, Problems of Information Transmission, 6 (1973), pp. 336-348.

[2] L. M. BREGMAN, Certain properties of nonnegative matrices and their permanents, Soviet. Math. Dokl., 14 (1973), pp. 945-949.

[3] H. Minc, Upper bounds for permanents of (0, 1)-matrices, Bull. Amer. Math. Soc., 69 (1963), pp. 789-791.

[4] N. PIPPENGER, The complexity of seldom-blocking networks, IEEE Internat. Comm. Conf., 12 (1976), pp. 7-8-7-12.

[5] N. PipPenger AND L. G. VAliant, Shifting graphs and their applications, J. Assoc. Comput. Mach., 23 (1976), pp. 423-432.

[6] A. Schrijver, A short proof of Minc's conjecture, J. Combinatorial Theory (A), 25 (1978), pp. 80-83.

[7] J. StiRling, Methodus Differentialis, London, 1730. 